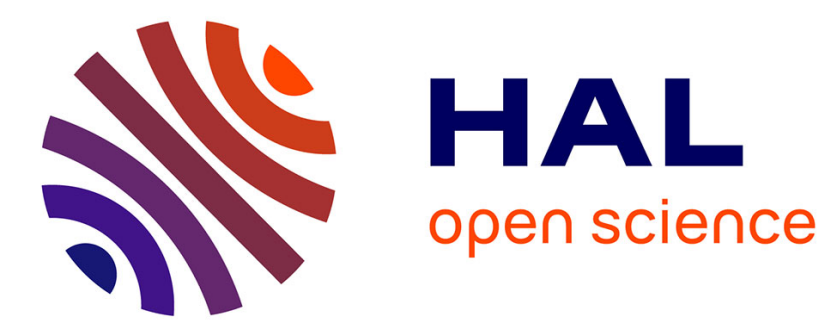

\title{
Ion Sound Solitary Waves with Density Depressions
}

\author{
R. Cairns, R. Bingham, R. Dendy, C. Nairn, P. Shukla, A. Mamun
}

\section{To cite this version:}

R. Cairns, R. Bingham, R. Dendy, C. Nairn, P. Shukla, et al.. Ion Sound Solitary Waves with Density Depressions. Journal de Physique IV Proceedings, 1995, 05 (C6), pp.C6-43-C6-48. 10.1051/jp4:1995608 . jpa-00253971

\section{HAL Id: jpa-00253971 https://hal.science/jpa-00253971}

Submitted on 1 Jan 1995

HAL is a multi-disciplinary open access archive for the deposit and dissemination of scientific research documents, whether they are published or not. The documents may come from teaching and research institutions in France or abroad, or from public or private research centers.
L'archive ouverte pluridisciplinaire HAL, est destinée au dépôt et à la diffusion de documents scientifiques de niveau recherche, publiés ou non, émanant des établissements d'enseignement et de recherche français ou étrangers, des laboratoires publics ou privés. 


\title{
Ion Sound Solitary Waves with Density Depressions
}

\author{
R.A. Cairns, R. Bingham*, R.O. Dendy**, C.M.C. Nairn***, P.K. Shukla**** and A.A. Mamun \\ School of Mathematical and Computational Sciences, University of St Andrews, North Haugh, \\ St Andrews, Fife, KY16 9SS, U.K. \\ * Space Science Department, Rutherford Appleton Laboratory, Chilton, Didcot, Oxfordshire, OX11 OQX, \\ $U . K$. \\ ** UKAEA, Government Division, Fusion, Culham, Abingdon, Oxfordshire, OX14 3DB, U.K, \\ *** Swedish Institute of Space Physics, Box 812, 98128 Kiruna, Sweden \\ ****Institut für Theoretische Physik 4, Ruhr-Universität Bochum, 44780 Bochum, Germany
}

\begin{abstract}
We show that a non-thermal electron distribution may change the nature of ion acoustic solitons. If the ions are assumed to respond as a fluid to perturbations in the potential, with no significant trapping in a potential well, then a thermal plasma only supports a solitary waves with a density peak. However, with a suitable distribution of non-thermal particles, solitary waves with both density peaks and density depressions may coexist. This may have applications to magnetosperic observations, where solitary structures with lowered densities have been observed in regions where the electron distribution is also seen to be non-thermal.
\end{abstract}

\section{INTRODUCTION}

Observations made by the Freja and Viking spacecraft $[1,2]$ have shown solitary structures in the magnetosphere with density depressions, sometimes associated with an enhanced level of lower hybrid turbulence, in which case they may be explained as cavities produced by the ponderomotive force of the lower hybrid waves. However, in some cases the density depressions seem to exist without any associated turbulence. This latter class of events motivated the present study, in which it is shown that a nonthermal electron distribution may change the nature of ion acoustic solitary waves in such a way as to allow just such a structure. The structures we find involve only a simple fluid response of the ions and so differ fundamentally those found in some previous work on ion holes [3] which involve a Bernstein - Greene - Kruskal type of equilibrium in which trapped ions play an essential role while the electrons are assumed to be Maxwellian.

We begin by taking a particular example, in which the electron distribution has an enhanced population of energetic particles rather like that produced by quasilinear diffusion. Distributions of this type are observed in the magnetospere[4]. In a simple one dimensional theory, we show how the solitary wave structure changes. We then go on to discuss some more general criteria, by means of which an electron distribution function with a given parametric form can be analysed and the nature of 
small amplitude solitary waves predicted, and conclude with a brief discussion of three-dimensional structures.

\section{BASIC THEORY}

We look at a simple one dimensional problem in which it is assumed that some stationary structure exists and that in its rest frame the potential is $\phi(x)$. The ions will be taken to be cold. If the velocity of the solitary structure with respect to the ions is $V_{0}$, it follows in a straightforward way from the ion equation of motion and conservation of ion flux that the ion density is

$$
n_{i}=\frac{n_{0}}{\sqrt{1-\frac{2 e \phi}{m_{i} V_{0}^{2}}}}
$$

Here $n_{0}$ is the density outside the structure and, for simplicity, the ions are assumed singly charged so that this is also the electron density far from the solitary wave.

To model an electron distribution with a population of fast particles we take it to be of the form

$$
f_{e}(v)=\frac{n_{0}}{(3 \alpha+1) \sqrt{2 \pi v_{e}^{2}}}\left(1+\frac{\alpha v^{4}}{v_{e}^{4}}\right) \exp \left(-\frac{v^{2}}{2 v_{e}^{2}}\right) .
$$

This distribution is shown in Fig. 1 for $\alpha=0.2$, a value which we shall continue to use in numerical illustrations. The electron distribution shown in Fig.1 is of a shape which might be expected if particles were being accelerated by quasilinear diffusion as a result of a spectrum of waves with phase velocities in the region where the distribution has shoulders.

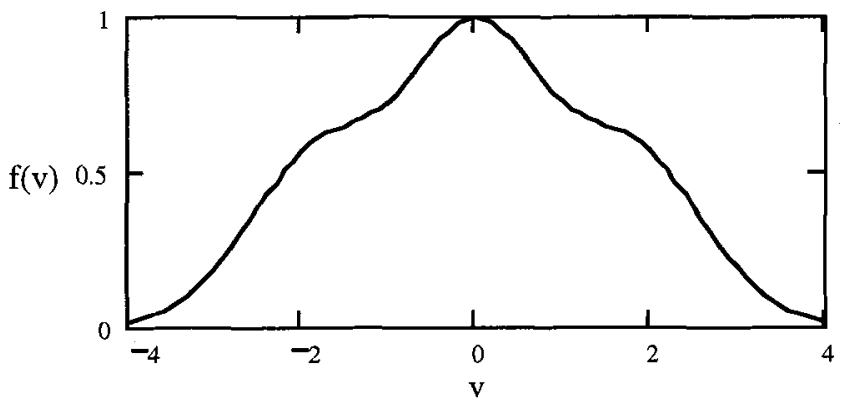

Fig. 1 The electron distribution function used to illustrate the possibility of rarefactive solitary waves.

We now assume that the velocity of the solitary wave is small compared to the electron thermal velocity and that the electrons take up an equilibrium distribution which is a function of their energy and is given simply by replacing $v^{2}$ in (2) with $v^{2}-\frac{2 e \phi}{m_{e}}$. It is convenient to introduce a dimensionless potential $\Phi=\frac{e \phi}{m_{e} v_{e}^{2}}$, so that when we integrate over velocity to find the electron density the result is

We also introduce the Mach number

$$
n_{e}=n_{0} \exp (\Phi) \frac{4 \alpha \Phi^{2}-4 \alpha \Phi+3 \alpha+1}{3 \alpha+1} .
$$

$$
M=\frac{V_{0}}{\sqrt{\frac{m_{e} v_{e}^{2}}{m_{i}}}} .
$$


Note that this is the true Mach number relative to the propagation velocity of small amplitude ion acoustic waves only if $\alpha=0$, since the wave velocity is greater in the presence of non-thermal particles.

We now use (2) and (3) in Poisson's equation. With lengths defined in terms of $v_{e} / \omega_{p e}$, ie the Debye length determined by the thermal component of the electrons, the result is

$$
\frac{d^{2} \Phi}{d x^{2}}=-\frac{1}{\sqrt{1-\frac{2 \Phi}{M^{2}}}}+\exp (\Phi) \frac{4 \alpha \Phi^{2}-4 \alpha \Phi+3 \alpha+1}{3 \alpha+1} .
$$

The qualitative nature of the solutions of this is most easily seen by introducing the Sagdeev potential or pseudopotential [5], defined so that (4) takes the form

$$
\frac{d^{2} \Phi}{d x^{2}}=-\frac{d V(\Phi)}{d \Phi}
$$

The problem is then analogous to particle motion in a potential $V$. The condition for existence of a solitary wave is that the origin be an unstable point and there be a potential well so that the "particle" which leaves the origin as $x \rightarrow-\infty$ is reflected and returns to the origin as $x \rightarrow+\infty$. In more technical terms, the unstable fixed point at the origin of the phase space of (5) should have a homoclinic orbit. If $\alpha=0$ the origin is a stable fixed point for Mach numbers less than one, and as $M$ goes above one, a potential well develops on the positive $\Phi$ side, giving solitary waves with a positive density perturbation. In this case there is never a potential well on the negative side.

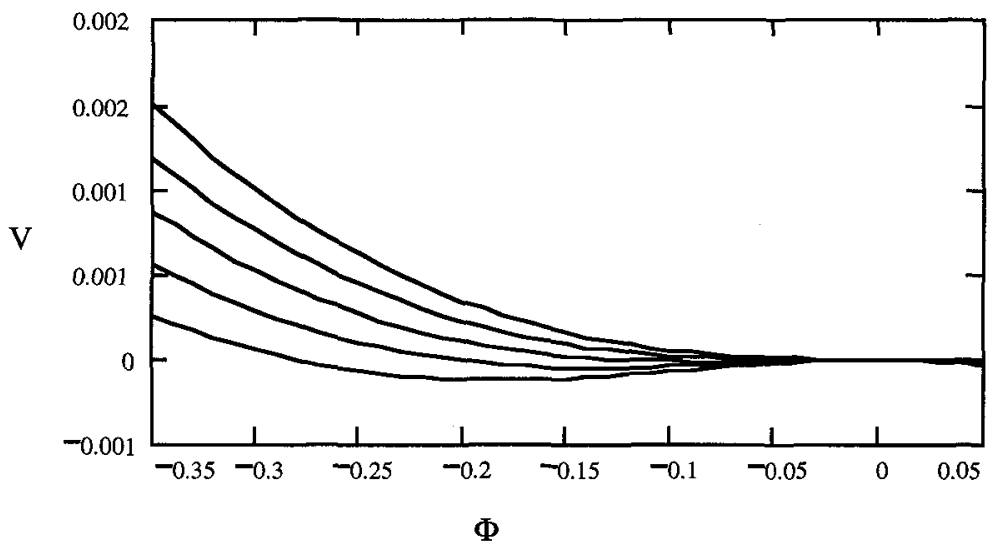

Fig.2 The Sagdeev potential for a series of Mach numbers, 1.41 (top) in steps of .01 to 1.45 (bottom).

If we take $\alpha=0.2$ then the behaviour changes and now a potential well appears on the negative side, as shown in Fig.2. With our definition of the Mach number, the critical value above which small amplitude oscillatory solutions go over to solitary wave solutions is $\sqrt{2}$ rather than 1. A different scale view of the potential, shown in Fig.3, shows that there is a deeper potential well on the positive side. Thus, positive and negative solitary waves can coexist, with the positive wave being of much greater amplitude when we are just above the critical Mach number, as illustrated in Fig. 4 .

\section{CRITERIA FOR NATURE OF SOLITONS}

Some more general results can be obtained as follows. The nature of those solitons whose amplitude tends to zero as the Mach number tends to its critical value can be 


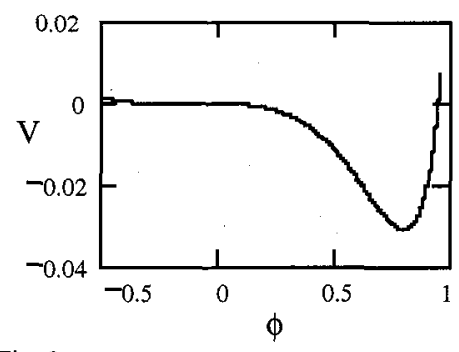

Fig. 3 The Sagdeev potential for $M=1.45$, scaled to show the potential well for $x>0$.

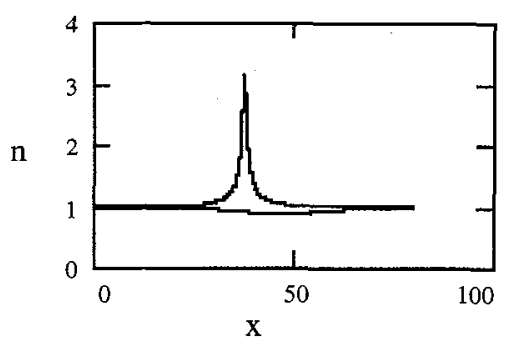

Fig.4 Positive and negative solitary waves for $\mathrm{M}=1.45$.

found by expanding the Sagdeev potential to third order in a Taylor series in $\phi$. The critical Mach number is that which corresponds to the vanishing of the quadratic term. If at the same time the cubic term is negative, then there is a potential well on the negative side and if the cubic term is positive, there is a potential well on the positive side. An expansion to third order of this sort corresponds, of course, to taking the equation of motion to second order. This is the order in which reductive perturbation schemes lead to a Korteweg-de Vries equation and it is easily shown that eqn. (4), when approximated to this order, leads to the familiar $\operatorname{sech}^{2}$ profile which is the steady state solution of the $\mathrm{KdV}$ equation. The co-existence of two types of soliton, as shown in Fig.3, is, of course, only predicted if we go to higher order.

For our particular distribution function, the expansion of the Sagdeev potential around the origin is

$$
V(\Phi)=\left(\frac{\alpha-1}{6 \alpha+2}+\frac{1}{2 M^{2}}\right) \Phi^{2}+\left(\frac{1}{2 M^{4}}-\frac{1}{6}\right) \Phi^{3}+\ldots \ldots . .
$$

From this we see that the critical Mach number at which the second derivative changes sign is given by

$$
M^{2}=\frac{3 \alpha+1}{1-\alpha}
$$

and at this value the third derivative is negative if $\alpha>\frac{\sqrt{3}-1}{3+\sqrt{3}}=0.155$. Thus we obtain, in a simple way, a lower bound for the energetic population in our particular class of distributions in order that negative solitons may exist.

Another example which is a popular model in various applications and has been considered previously by other workers [6] is the double Maxwellian

$$
f(v)=\alpha \frac{n_{0}}{\sqrt{2 \pi}} \exp \left(-\frac{v^{2}}{2}\right)+(1-\alpha) \frac{n_{0}}{\sqrt{2 \pi V^{2}}} \exp \left(-\frac{v^{2}}{2 V^{2}}\right)
$$

where we have normalised to the thermal velocity of the first component. The electron density, with the potential normalised as before, is

$$
n_{e}=n_{0} \alpha \exp (\Phi)+n_{0}(1-\alpha) \exp \left(\frac{\Phi}{V^{2}}\right) .
$$

Taking the ion contribution as before we find, on expanding in a Taylor series, that the quadratic term becomes negative if

$$
M^{2}>\frac{V^{2}}{\alpha V^{2}+1-\alpha}
$$

and that the third order coefficient is negative at the critical Mach number if

$$
\frac{1}{6} \frac{V^{2}-5-\sqrt{V^{4}-10 V^{2}+1}}{V^{2}-1}<\alpha<\frac{1}{6} \frac{V^{2}-5+\sqrt{V^{4}-10 V^{2}+1}}{V^{2}-1} .
$$


This only makes sense if the quantity under the square root sign is positive, which imposes the restriction that $V^{2}$ be between 0 and 0.101 or greater than 9.899 . The two ranges reflect an obvious symmetry in the problem, where replacing $V 2$ with its inverse and $\alpha$ with 1- $\alpha$ gives the same form of distribution function. In the regime where small amplitude negative solitons exist, examination of the Sagdeev potential shows that larger amplitude positive solitons may also exist, as in our earlier example.

\section{THREE DIMENSIONAL STRUCTURES}

A very simple three dimensional analogue of these structures can be constucted by assuming that they are moving parallel to a strong magnetic field. If the ion Larmor radius is small compared to the size of the structure we can just consider the ions to be a beam flowing along the field lines (in the rest frame of the structure ). The density then just depends on the potential as before. We assume also that the electrons have the same kind of adiabatic response and that the one dimensional distribution considered up to now is obtained by integrating over the parallel degrees of freedom. In this case, the only change is that in Eqn. (4) $\frac{d^{2} \Phi}{d x^{2}}$ is replaced with $\nabla^{2} \Phi$. Spherically symmetrical solitary structures exist as solutions of this equation and an example, for the distribution function given by Eqn. (2) is illustrated below.

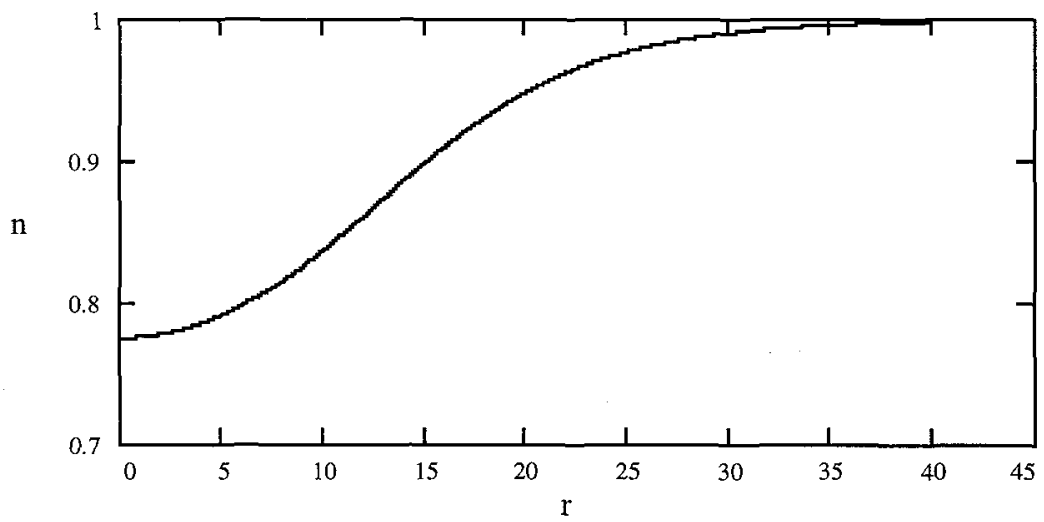

Fig.5 Ion density as a function of radius for a spherically symmetrical structure.

\section{CONCLUSIONS}

We have shown that the structure of ion acoustic type solitary waves in a plasma can be modified in the presence of non-thermal electron distributions. If there are enough particles in an energetic tail then the standard compressive soliton found by small amplitude expansion techniques is changed into a rarefactive soliton. An analysis valid for arbitrary amplitude shows that in many cases a large amplitude compressive solitary wave can co-exist with the smaller amplitude rarefactive wave. Although we have assumed an electron distribution function which is a symmetrical function of velocity, this is not necessary. It is clear that doubling the hot electron component while confining it to one side of the distribution gives precisely the same electron density.

This analysis may be of relevance to observations in the magnetosphere of density depressions. A possible scenario is that lower hybrid turbulence produces, through modulational instability, cavities which collapse until the lower hybrid wave amplitude is sufficient to trap and accelerate a substantial number of electrons $[7,8]$. The damping of the turbulence could then leave a cavity and also create just the kind 
of energetic electron population necessary for it to live on as an ion acoustic solitary structure no longer supported by the ponderomotive pressure of the high frequency turbulence. However, the type of electron distribution we have looked at is common to many space and laboratory plasmas in which wave damping produces an electron tail, so the theory may be of more general interest.

\section{Acknowledgements}

This research was supported by the European Commission through Grant C.E.C. -CHRX-CT93-0356. A.A. Mamun was supported by a Commonwealth Scholarship.

\section{References}

[1] R. Bostrom et al, Phys.Rev. Lett. 61 (1988) 82-85.

[2] P.O. Dovner et al, . Geophys.Res. Lett. 21 (1994) 1827-1830.

[3] H. Schamel, Phys. Reports 140 (1986) 161-191.

[4] D.S. Hall et al, J. Geophys. Res. 96 (1991) 7869-7891.

[5] R.Z. Sagdeev, in Reviews of Plasma Physics, Vol.4, ed M A Leontovich (Consultants Bureau, New York, 1966).

[6] K. Nishihara and M. Tajiri, J. Phys. Soc. Japan 50 (1981) 4047-4053.

[7] V.D. Shapiro et al, Phys. Plasmas 2 (1995) 3148-3162.

[8] R. Bingham et al, these Proceedings. 